Research Paper

\title{
Comparison of RECIST 1.0 and RECIST 1.1 in Patients with Metastatic Cancer: A Pooled Analysis
}

\author{
Jung Han Kim ${ }^{1 * 凶}$, Seon Jeong Min² ${ }^{*}$, Hyun Joo Jang ${ }^{3}$, Ji Woong Cho ${ }^{4}$, Soo Ho Kim¹, Hyeong Su Kim ${ }^{1}$
}

1. Department of Internal Medicine, Kangnam Sacred Heart Hospital, Hallym University Medical Center, Hallym University College of Medicine, Seoul 150-950, Republic of Korea

2. Department of Radiology, Dongtan Sacred Heart Hospital, Hallym University Medical Center, Hallym University College of Medicine, Hwasung 445-907, Republic of Korea

3. Department of Internal Medicine, Dongtan Sacred Heart Hospital, Hallym University Medical Center, Hallym University College of Medicine, Hwasung 445-907, Republic of Korea

4. Department of Surgery, Hallym University Sacred Heart Hospital, Hallym University Medical Center, Hallym University College of Medicine, Anyang 431-070, Republic of Korea

* Jung Han Kim and Seon Jeong Min equally contributed as to this study.

$\triangle$ Corresponding author: Jung Han Kim, MD. PhD., Department of Internal Medicine, Kangnam Sacred-Heart Hospital, Hallym University Medical Center, Shingilro 1, Youngdeungpo-Gu, Seoul 150-950, South Korea. E-mail address: harricil@hotmail.com, harricil@hallym.or.kr

(C) 2015 Ivyspring International Publisher. Reproduction is permitted for personal, noncommercial use, provided that the article is in whole, unmodified, and properly cited. See http://ivyspring.com/terms for terms and conditions.

Received: 2014.12.11; Accepted: 2015.01.21; Published: 2015.02.25

\begin{abstract}
Background: We conducted this pooled analysis to investigate the impact of RECIST 1.1 on the selection of target lesions and classification of tumor response, in comparison with RECIST 1.0.

Methods: We searched MEDLINE and EMBASE for articles with terms of RECIST 1.0 or RECIST 1.1. We looked into all abstracts and virtual meeting presentations from the conferences of ASCO and ESMO between 2009 and 2013.

Results: There were six articles in the literature comparing the clinical impacts of RECIST 1.0 and RECIST 1.1 in patients with metastatic cancer. A total of 359 patients were recruited from the six trials; 217 with non-small cell lung cancer, 61 with gastric cancer, 58 with colorectal cancer, and 23 with thyroid cancer. The number of target lesions by RECIST 1.1 was significantly lower than that by RECIST $1.0(P<0.001)$. Because of new lymph node criteria, fourteen patients $(3.1 \%)$ had no target lesions when adopting RECIST 1.1. RECIST 1.1 showed high concordance with RECIST 1.0 in the assessment of tumor responses $(\mathrm{k}=0.903)$. Sixteen patients $(4.8 \%)$ showed disagreement between the two criteria.

Conclusion: This pooled study demonstrated that RECIST 1.1 showed a highly concordant response assessment with RECIST 1.0 in patients with metastatic cancer.
\end{abstract}

Key words: RECIST 1.0; RECIST 1.1; Target lesion; Tumor response

\section{Introduction}

The decision on subsequent cancer treatments usually depends on radiologic changes in the tumor burden, so the accurate assessment of objective therapeutic response is essential for patients receiving anti-cancer treatments. Since the World Health Organization (WHO) issued objective response criteria in 1979, the WHO guidelines have been used as the standard method for evaluating tumor response [1]. Tumor sizes are measured bi-dimensionally by the product of the longest diameter and its longest perpendicular diameter for each tumor, and tumor responses are expressed as percentage changes in the sum of tumor measurements from baseline. Because the methods for selecting and measuring target le- 
sions were not clearly described in the WHO guidelines, however, the assessment of tumor response has been poorly reproducible between investigators [2,3]. In clinical practice, measuring with two dimensions and then calculating the sums of their products not only are laborious but also has a potential risk of errors. Theoretically, the simple sum of the maximum diameters of target lesions is more linearly related to cells killed than the sum of the bi-dimensional products [4]. Furthermore, the recent development of new classes of anti-cancer agents and new imaging technologies have necessitated a new methodology for evaluating tumor response $[5,6]$.

In 2000, the Response Evaluation Criteria in Solid Tumors (RECIST) Working Group introduced a new set of tumor response criteria, the RECIST guidelines version 1.0 (RECIST 1.0) [7]. RECIST 1.0 adopted uni-dimensional measurement, instead of the bi-dimensional criterion in the WHO guidelines. Other important features of RECIST 1.0 included definition of minimum size of measurable lesion by computed tomography (CT) and instruction on how many lesions to be evaluated (up to ten, with a maximum of five per organ). RECIST 1.0 had been widely accepted as the standardized method for tumor response assessment, particularly in oncologic trials with primary end point of objective response or time to progression. However, a number of questions and issues were raised, which included the number of target lesions and the size of lymph nodes (LNs) to be measured. Subsequent rapid innovation of new imaging technologies, such as multi-detector computed tomography (MDCT) and positron emission tomog- raphy (PET), requested an update of RECIST 1.0 [8].

In 2009, the RECIST Working Group published a revised version of RECIST guidelines (RECIST 1.1) [9], which was based partly on the analyses of the database of about 6,500 patients with more than 18,000 target lesions from 16 clinical trials [10-12]. The most important changes in RECIST 1.1 include reduction in the maximum number of target lesions (up to five in total, with two per organ), new criteria for LN measurement, augmented definition of progressive disease (PD), new criteria for selecting bone lesions and cysts as target lesions, and the inclusion of PET findings for assessing tumor response (Table 1) [9,13-15].

With the expectation of improving feasibility through a more convenient and accurate assessment of both tumor response and time to progression, investigators have started to adopt RECIST 1.1 in clinical trials. Since being introduced into clinical practice, RECIST 1.1 have shown high concordance with RECIST 1.0 in the assessment of tumor responses for patients with advanced or metastatic non-small cell lung cancer (NSCLC) [16-18], gastric cancer (AGC) [19], colorectal cancer (CRC) [20], and thyroid cancer (TC) [21]. However, each study had a small number of patients with a single type of primary cancer, so it is still necessary to reveal how RECIST 1.1 affects the selection and measurement of target lesions and assessment of tumor responses in patients with metastatic cancer.

We conducted this pooled analysis to investigate the impact of RECIST 1.1 on the selection of target lesions and classification of tumor response, in comparison with RECIST 1.0.

Table 1. Summary of the major changes between RECIST 1.0 and RECIST 1.1 [15]

\begin{tabular}{|c|c|c|}
\hline & RECIST 1.1 & RECIST 1.0 \\
\hline Number of target lesions & Up to 2 per organ; up to 5 in total & Up to 5 per organ; up to 10 in total \\
\hline Minimum size of target lesions & $\begin{array}{l}10 \mathrm{~mm} \text { when slice thickness of CT is } \leq 5 \mathrm{~mm} \text {, or } 2 x \text { slice } \\
\text { thickness when it slice thickness is } \geq 5 \mathrm{~mm}\end{array}$ & $10 \mathrm{~mm}$ (spiral CT) or $20 \mathrm{~mm}$ (non-spiral CT) \\
\hline Assessment of lymph nodes & $\begin{array}{l}\text { Short-axis measurements should be used; } \\
\geq 15 \mathrm{~mm} \text { for target } \\
\geq 10 \mathrm{~mm} \text { to }<15 \mathrm{~mm} \text { for non-target }<10 \mathrm{~mm} \text { for } \\
\text { non-pathological } \\
\text { Lymph node }<10 \mathrm{~mm} \text { in shot axis is } \mathrm{CR}\end{array}$ & $10 \mathrm{~mm}$ in long axis for target \\
\hline CR of lymph nodes & $\begin{array}{l}\text { May be used as target lesions } \\
\text { (special notes) }\end{array}$ & Not specified \\
\hline Bone lesions and cysts & $5 \mathrm{~mm}$ absolute increase is required & $\begin{array}{l}\text { Non-measurable } \\
\text { (no specification) }\end{array}$ \\
\hline PD of target lesions & $\begin{array}{l}\text { Increase of non-target lesions is PD only if the increase is } \\
\text { representative of substantial change in tumor burden }\end{array}$ & No minimum absolute size increase is required \\
\hline PD of non-target lesions & Included only in the detection of new lesions & $\begin{array}{l}\text { Increase in size of one or a few non-target lesions is } \\
\text { regarded as PD, even when target lesions are stable } \\
\text { or responding. }\end{array}$ \\
\hline PET scan & & Not included \\
\hline
\end{tabular}




\section{Materials and methods}

\section{Searching strategy}

We searched the Cochrane Central Register of Controlled Trials (CENTRAL, Issue 5 of 12, May 2014), MEDLINE (from 2009 to May week 4, 2014) and EMBASE (from 2009 to week 20, 2014) for articles that included the following terms in their titles, abstracts, or keywords; 'RECIST 1.0 or RECIST 1.1', 'comparison', 'target lesion' and 'tumor response'. In addition, we surveyed all the references of relevant articles and reviews and used the 'related articles' feature in PubMed to identify the related articles. We also searched all abstracts and virtual meeting presentations from the conferences of the American Society of Clinical Oncology and European Society for Medical Oncology held between 2009 and 2013.

We thoroughly looked into all potentially eligible studies which were indentified via the above searching strategy. Clinical studies comparing the assessment of tumor response using RECIST 1.0 and RECIST 1.1 in patients who were treated with cytotoxic agents or target agents were included in the meta-analysis.

\section{Statistical analyses}

A paired Student's $t$ test was used to assess the statistical significance of changes in the number of target lesions between RECIST 1.0 and RECIST 1.1. Chi-square test was used to compare the overall response rates (ORRs) between two groups. P-values less than 0.05 were considered significant. The level of concordance of the best tumor responses between two criteria was assessed using карра statistics. A kappa value of more than 0.75 was interpreted as showing excellent agreement.

\section{Results}

\section{Eligible studies}

There were seven articles [16-22] and one abstract [23] in the literature comparing the clinical impacts of RECIST 1.0 and RECIST 1.1 in patients with solid tumors. However, the abstract [23] and one article [22] compared the two criteria mainly focused on the measurement of the LNs, with little information about concordance of tumor responses. Finally, six studies [16-21] that investigated the concordance of tumor responses between RECIST 1.0 and RECIST 1.1 were selected.

\section{Patients' characteristics}

A total of 359 patients with metastatic cancer were recruited from the six trials; 217 with NSCLC [16-18], 61 with GC [19], 58 with CRC [20], and 23 with
TC [21]. The characteristics and clinical features of the patients were briefly described in Table 2. However, two trials by Sun et al. [16] and Nishino et al. [17] had no enough basic information about the enrolled patients.

Most patients (97.2\%) had at least one target lesion according to RECIST 1.0. However, 11 patients (3.1\%) had no target lesions when RECIST 1.1 was used. The most common metastatic site with measurable target lesions in patients with GC or CRC was the LNs, followed by the liver.

Patients with metastatic NSCLC were all treated with epidermal growth factor tyrosine kinase inhibitors (EGFR-TKI) such as gefitinib and elrotinib. Patients with metastatic GC or CRC received a first-line chemotherapy, most commonly with FOLFOX (5-fluorouracil/leucovorin plus oxaliplatin). Patients with radioactive iodine-refractory TC were treated with sorafenib, an oral, small molecule TKI.

\section{Number of target lesions}

The data about the number of target lesions was available in five studies [17-21], except for the trial by Sun et al. Especially for the two studies $[19,20]$, we also used the raw data because the studies had been conducted in our institution (Hallym University Medical Center). The number of target lesions according to RECIST 1.1 was significantly lower than that according to RECIST 1.0 ( $\mathrm{P}<0.001$, paired Student's $t$-test). The median number of target lesions was 3 (range, 0-10) by RECIST 1.0 and 2 (range, 0-5) by RECIST 1.1, respectively. Among 255 patients from the 5 studies, $157(61.6 \%)$ showed a decrease in the number of target lesions when RECIST 1.1 was used. In 49 patients $(21.8 \%)$, the decreased total number of target lesions was resulted from the reduced maximum number of target lesion per organ in RECIST 1.1. Twenty-six patients $(11.6 \%)$ showed a decrease in the number of target lesions due to both the new LN criteria and the reduction of maximum target lesions. The new LN criteria of RECIST 1.1 contributed to the reduction of target lesions in 82 patients (32.2\%). Among 359 patients, $14(3.1 \%)$ had no target lesions when adopting RECIST 1.1, because all their target lesions were LNs < $15 \mathrm{~mm}$ along the short axis.

\section{Re-categorization of LNs by RECIST 1.1}

The data about re-categorization of LNs by RECIST 1.1 that were candidate target lesions based on RECIST 1.0 was only described in the study of metastatic CRC by Jang et al. [20]. From 58 patients, a total of $95 \mathrm{LNs}$ were regarded as target lesions according to RECIST 1.0. According to RECIST 1.1, however, only $40 \%$ of the LNs were classified as target lesions. 
Table 2. Summary of the 6 studies comparing RECIST 1.0 and RECIST 1.1

\begin{tabular}{|c|c|c|c|c|c|c|}
\hline Characteristics & $\begin{array}{l}\text { Sun et al. [16] } \\
\text { NSCLC } \\
(\mathrm{n}=104)\end{array}$ & $\begin{array}{l}\text { Nishino et al. [17] } \\
\text { NSCLC } \\
(\mathrm{n}=43)\end{array}$ & $\begin{array}{l}\text { Nishino et al. [18] } \\
\text { NSCLC } \\
(\mathrm{n}=70)\end{array}$ & $\begin{array}{l}\text { Jang et al. [19] } \\
\text { GC } \\
(\mathrm{n}=61)\end{array}$ & $\begin{array}{l}\text { Jang et al. [20] } \\
\text { CRC } \\
(n=58)\end{array}$ & $\begin{array}{l}\text { Ruan et al. [21] } \\
\text { TC } \\
(n=23)\end{array}$ \\
\hline & no. of pts & no. of pts & no. of pts & no. of pts & no. of pts & no. of pts \\
\hline $\begin{array}{l}\text { Age, years } \\
\text { (range) }\end{array}$ & na & na & $\begin{array}{l}\text { median } 62 \\
(35-84)\end{array}$ & $\begin{array}{l}\text { median } 58 \\
(26-78)\end{array}$ & $\begin{array}{c}\text { median } 62 \\
(42-79)\end{array}$ & $\begin{array}{l}\text { mean 54 } \\
(33-75)\end{array}$ \\
\hline Gender & na & na & & & & \\
\hline Male & & & $12(17.1 \%)$ & $42(68.9 \%)$ & $29(50 \%)$ & $14(60.9 \%)$ \\
\hline Female & & & $58(82.9 \%)$ & $19(31.1 \%)$ & $29(50 \%)$ & $9(39.1 \%)$ \\
\hline Histology & na & na & & & & \\
\hline Adenocarcinoma & & & $63(90 \%)$ & $61(100 \%)$ & $58(100 \%)$ & - \\
\hline Well/moderately differentiated & & & na & $22(36.1 \%)$ & $35(60.3 \%)$ & - \\
\hline Poorly differentiated & & & na & $39(63.9 \%)$ & $23(39.7 \%)$ & - \\
\hline Non-adneocarcinoma & & & $7(10 \%)$ & 0 & 0 & - \\
\hline Papillary & & & - & - & - & $22(95.6 \%)$ \\
\hline Follicular & & & - & - & - & $1(4.4 \%)$ \\
\hline Target lesions by RECIST 1.0 & $104(100 \%)$ & $43(100 \%)$ & $69(98.6 \%)$ & $61(100 \%)$ & $58(100 \%)$ & $14(60.9 \%)$ \\
\hline Lungs & na & na & na & $2(3.2 \%)$ & $8(13.8 \%)$ & 0 \\
\hline Lymph nodes & na & na & na & $51(83.6 \%)$ & $37(63.8 \%)$ & 0 \\
\hline Liver & na & na & na & $8(13.1 \%)$ & $27(46.5 \%)$ & 0 \\
\hline Adrenal glands & na & na & na & 0 & $2(3.4 \%)$ & 0 \\
\hline Ovary & na & na & na & 0 & $3(5.2 \%)$ & 0 \\
\hline Median target lesions*(range) & na & $2(1-9)$ & $2(1-10)$ & $3(1-10)$ & $4(1-10)$ & $3(1-6)$ \\
\hline No target lesion by RECIST 1.1 & 0 & $3(6.9 \%)$ & $2(2.9 \%)$ & $3(4.9 \%)$ & $6(10.3 \%)$ & 0 \\
\hline PET & 0 & $6(4.3 \%)$ & $10(14.3 \%)$ & 0 & 0 & $5(21.7 \%)$ \\
\hline \multicolumn{7}{|l|}{ Treatment } \\
\hline Erlotinib & $36(34.6 \%)$ & $43(100 \%)$ & $63(90 \%)$ & 0 & 0 & 0 \\
\hline Gefitinib & $68(65.4 \%)$ & 0 & $7(10 \%)$ & 0 & 0 & 0 \\
\hline Capecitabine + cisplatin & 0 & 0 & 0 & $21(34.4 \%)$ & 0 & 0 \\
\hline FOLFOX & 0 & 0 & 0 & $40(65.6 \%)$ & $53(91.4 \%)$ & 0 \\
\hline FOLFIRI & 0 & 0 & 0 & 0 & $5(8.6 \%)$ & 0 \\
\hline Sorafenib & 0 & 0 & 0 & 0 & 0 & $23(100 \%)$ \\
\hline
\end{tabular}

Abbreviations: NSCLC, non-small cell lung cancer; GC, gastric cancer; CRC, colorectal cancer; TC, thyroid cancer;

na, not available; no. of pts, number of patients; PET, positron emission tomography;

FOLFOX, oxaliplatin plus 5-fluououracil/leucovorin; FOLFIRI, Irinotecan plus 5-fluorouracil/leucovorin.

* according to RECIST 1.0.

\section{Tumor responses}

We compared the tumor responses between the two criteria using 332 patients who had at least one target lesion based on RECIST 1.1. The remaining 27 patients were excluded from the comparison because they had no target lesions according to RECIST 1.1 and the tumor responses were uncertain in most of them. The results are presented in Table 3. There was high concordance between RECIST 1.0 and RECIST 1.1 in the assessment of tumor responses. The estimated kappa value was 0.903 , with $95 \%$ confidence interval of $0.863-0.943$. When we compared the ORRs, which were estimated regardless of the primary site and anti-cancer treatment, were not significantly different between the two criteria $(42.2 \%$ by RECIST 1.1 versus $39.1 \%$ by RECIST $1.0, \mathrm{P}=0.430$ ).

A total of 16 patients $(4.8 \%)$ showed disagreement between the two criteria. The details of the patients showing disagreement between RECIST 1.0 and RECIST 1.1 were described according to reference in Table 4. The discrepancies of the two criteria were between PR and SD in 8 patients, SD and PD in 6, and $\mathrm{PR}$ and $\mathrm{CR}$ in 2. No patients showed disagreement between PR and PD. The most common cause of the discordance was the new LN criteria, which led to the different response classification in $9(56.3 \%)$. Four patients $(25.0 \%)$ showed disagreement between the two criteria because of the maximum of target lesions ( 5 in total, with up to 2 lesions per organ) in RECIST 1.1. Two patients with SD according to RECIST 1.0 were reclassified as PD because of the new lesions noted on PET/CT.

Table 3. Comparison of tumor responses by RECIST 1.0 versus RECIST 1.1

\begin{tabular}{llllll}
\hline $\begin{array}{l}\text { Tumor response } \\
\text { by RECIST 1.0 }\end{array}$ & \multicolumn{5}{c}{ Tumor response by RECIST 1.1 } \\
\cline { 2 - 5 } & CR & PR & SD & PD & Total \\
\hline CR & 1 & 0 & 0 & 0 & 1 \\
PR & 2 & 125 & 2 & 0 & 129 \\
SD & 0 & 12 & 111 & 4 & 127 \\
PD & 0 & 0 & 3 & 72 & 75 \\
Total & 3 & 137 & 116 & 76 & 332 \\
\hline
\end{tabular}

Abbreviations: CR, complete response; PR, partial response; SD, stable disease; $\mathrm{PD}$, progressive disease

The level of concordance of tumor responses between RECISI 1.1 and RECIST 1.0 is 0.903 (95\% CI, 0.863-0.943).

The overall response rates were not significantly different between the two criteria ( $42.2 \%$ by RECIST 1.1 versus $39.1 \%$ by RECIST $1.0, \mathrm{P}=0.430$ ) 
Table 4. Summary of the patients showing disagreement between RECIST 1.0 and RECIST 1.1

\begin{tabular}{|c|c|c|c|c|c|}
\hline \multirow[t]{2}{*}{ Reference } & \multirow{2}{*}{$\begin{array}{l}\text { Tumor } \\
\text { type }\end{array}$} & \multicolumn{2}{|c|}{ Tumor response } & \multirow[t]{2}{*}{ No. of patients } & \multirow[t]{2}{*}{ Causes of disagreement } \\
\hline & & RECIST 1.0 & RECIST 1.1 & & \\
\hline \multirow[t]{3}{*}{ Sun et al. [16] } & \multirow[t]{3}{*}{ NSCLC } & PR & CR & 2 & LNs $<10 \mathrm{~mm}$ \\
\hline & & SD & PR & 3 & Equivocal LNs \\
\hline & & SD & PD & 1 & A definitely increased LN \\
\hline \multirow[t]{2}{*}{ Nishino et al. [17] } & \multirow[t]{2}{*}{ NSCLC } & SD & PD & 2 & New lesions on PET \\
\hline & & PD & SD & 1 & A single $\mathrm{LN}<10 \mathrm{~mm}$ \\
\hline Nishino et al. [18] & NSCLC & SD & PR & 1 & Decreased number of target lesion \\
\hline \multirow[t]{3}{*}{ Jang et al. [19] } & \multirow[t]{3}{*}{ GC } & PR & SD & 1 & Four LNs $<15 \mathrm{~mm}$ \\
\hline & & SD & PR & 1 & Up to 2 target lesion per organ \\
\hline & & SD & PD & 1 & Up to 2 target lesion per organ \\
\hline \multirow[t]{3}{*}{ Jang et al. [20] } & \multirow[t]{3}{*}{ CRC } & PR & SD & 1 & Two LNs $<15 \mathrm{~mm}$ \\
\hline & & PD & SD & 1 & Up to 2 target lesion per organ \\
\hline & & PD & SD & 1 & An absolute size increase of at least $5 \mathrm{~mm}$ \\
\hline Ruan et al. [21] & TC & SD & PR & 1 & Not described \\
\hline
\end{tabular}

\section{Discussion}

Since RECIST 1.1 was presented in 2009 [9], the impact of RECIST 1.1 has been compared with RECIST 1.0 in patients with metastatic NSCLC [16-18], AGC [19], CRC [20], and TC [21]. However, each study had a small number of patients with a single type of primary cancer. In this pooled study, we investigated the impact of RECIST 1.1 on the selection of target lesions and assessment of the best tumor responses. RECIST 1.1 significantly decreased the number of target lesions to be measured in patients with metastatic cancer. However, there was an excellent agreement in the assessment of tumor responses between RECIST 1.0 and RECIST 1.1.

As expected, RECIST 1.1 affected the number of target lesions. The maximum number of target lesions to be assessed in RECIST 1.1 is reduced from 10 to 5 in total, and from 5 to 2 per organ. While the total of 10 target lesions in RECIST 1.0 was arbitrarily selected, RECIST 1.1 defined a total of 5 lesions through the patients' data analysis [10] and statistical simulating studies [11,14]. Out of 255 patient from 5 studies in which the number of target lesions were described [17-21], 157 (61.6\%) showed a decrease in the number of target lesions when RECIST 1.1 was adopted. In 49 patients $(21.8 \%)$, the criteria of two lesions per organ contributed to the decreased number of target lesions. According to RECIST 1.1, lytic or mixed lytic-blastic bone lesions with an identifiable soft tissue component may be used as target lesions. In this pooled analysis with 359 patients, however, only one with TC newly had a bone target lesion when adopting RECIST 1.1.

RECIST 1.1 recommends the measurement of LN along its short axis, regarding LNs of at least $15 \mathrm{~mm}$ as target lesions. LN with at least $10 \mathrm{~mm}$ but less than 15 $\mathrm{mm}$ in its short axis, even though it may be pathological, is considered non-target lesion, and LN with a short axis of less than $10 \mathrm{~mm}$ is regarded as normal.
These changes in the LN evaluation criteria also had a considerable impact on the number of target lesions. In this meta-analysis, the new LN criteria of RECIST 1.1 led to the reduction of target lesions in 82 patients $(32.2 \%)$, including $26(11.6 \%)$ in whom the decrease was attributable to both the new LN criteria and the reduction of maximum target lesions.

From the RECIST data warehouse, $90.5 \%$ of LNs were regarded as target lesion according to the new LN criteria of RECIST 1.1 [13]. In the study of patients with GC by Jang et al., however, among 95 LNs considered to be target lesions by RECIST 1.0, only 38 (40 $\%)$ were defined as target lesions based on RECIST 1.1 [20]. These results are in agreement with those of the study conducted by Fuse at al. in patients with metastatic GC. Out of $172 \mathrm{LNs}$ regarded as target lesions by RECIST 1.0, only $66(38 \%)$ were defined as target lesions based on RECIST 1.1 [22]. Piatek et al. found the similar results in patients with prostate cancer. Among 158 LNs regarded as target lesions by RECIST 1.0, only $66(41.8 \%)$ satisfied the LN criteria of RECIST 1.1 [23]. Therefore, the new LN criteria of RECIST 1.1 may alter the eligibility of patients for clinical trials in which the ORR or time to progression is a primary endpoint. In the study by Fuse et al. the proportion of patients with target lesions was significantly decreased from $67 \%$ to $53 \%$ by adopting RECIST 1.1 [22]. In this meta-analysis, 14 patients (3.1\%) no longer had target lesions when adopting RECIST 1.1, because all their target lesions were LNs $<15 \mathrm{~mm}$ along the short axis. If studies using RECIST 1.1 had been planned, these patients would have been excluded from clinical trials. RECIST 1.1 with more stringent LN measurement rules, however, may categorize more patients as CR than RECIST 1.0. In the study by Sun et al., two NSCLC patients with PR according to RECIST 1.0 were re-categorized as CR because LNs with short axes of $<10 \mathrm{~mm}$ were considered normal based on RECIST 1.1 [16]. 
This pooled study demonstrates that there is high concordance between RECIST 1.0 and RECIST 1.1 in the assessment of tumor responses. When comparing the tumor response assessment in 332 patients who had at least one target lesion based on RECIST 1.1, the level of agreement in tumor responses between the two criteria was very high, with a kappa value of 0.903 . The ORRs estimated regardless of the primary site and anti-cancer treatment were not significantly different between the two criteria (42.2\% by RECIST 1.1 versus $39.1 \%$ by RECIST $1.0, P=0.430$ ). The disagreement between the two RECIST versions was observed in 16 patients (4.8\%). The most common cause of the discordance was the new LN criteria (9 patients), followed by the maximum of target lesions in RECIST 1.1 (6 patients). As patients who achieve PR or SD practically stay on the same treatment, patients showing discordance between PR and SD would have no significant clinical impact of RECIST 1.1. In this study, only six patients $(1.8 \%)$ displayed disagreement between SD and PD. Therefore, the clinical impact of RECIST 1.1 on changing therapeutic decisions seemed to be minimal.

Several limitations of this pooled analysis should be noted. First, PET was not routinely performed in all 6 studies. PET scans have an important role in the assessment of tumor response using RECIST 1.1. New lesions detected on PET scans change the tumor response from PR or SD according to RECIST 1.0 to PD according to RECIST 1.1. Therefore, the incorporation of PET may have a significant influence on the assessment of tumor responses based on RECIST 1.1. In this pooled analysis, only 21 patients $(5.8 \%)$ underwent PET. Two patients had new lesions on PET scans, which changed the tumor response from SD to PD. One patient with NSCLC did not undergo baseline PET, and a new lesion was detected on PET scans during therapy. The new lesion was confirmed by the follow-up CT. If the studies had performed PET more frequently, the newly detected lesions could have led to a lower concordance rate for tumor responses between the two RECIST versions. Second, the comparison of tumor responses between the two criteria was conducted only in patients with at least one target lesion according to RECIST 1.1. According to RECIST 1.0, the increase in size of one or a few non-target lesions was regarded as PD, even though target lesions are stable or responding. Based on RECIST 1.1, however, patients with PR or SD based on target lesion response are categorized as $\mathrm{PD}$, only if the increase of non-target lesions is representative of substantial change in tumor burden. Therefore, if the comparison had included patients with non-target lesion, the new criteria of non-target lesion would have affected the concordance between RECIST 1.0 and RECIST 1.1.
Third, this pooled analysis only contains patients with four types of primary tumors (NSCLC, GC, CRC, and TC). This means that the results may be insufficient to be generalized for patients with other primary cancer.

In conclusion, this pooled study demonstrates that RECIST 1.1 provides a highly concordant response assessment with RECIST 1.0 in patients with metastatic cancer. Because of the more stringent $\mathrm{LN}$ criteria, however, RECIST 1.1 may adversely affect the patients' eligibility for clinical trials.

\section{Conflict of Interest}

Authors do not have any conflict of interest.

\section{References}

1. Miller AB, Hoogstraten B, Staquet M, Winkler A. Reporting results of cancer treatment. Cancer. 1981;47:207-14.

2. Therasse P. Measuring the clinical response. What does it mean? Eur J Cancer. 2002;38:1817-23.

3. Choi JH, Ahn MJ, Rhim HC, Kim JW, Lee GH, Lee YY, et al. Comparison of WHO and RECIST criteria for response in metastatic colorectal cancer. Cancer Res Treat. 2005;37:290-3.

4. James K, Eisenhauer E, Christian M, Terenziani M, Vena D, Muldal A, Therasse $\mathrm{P}$, et al. Measuring response in solid tumors: unidimensional versus bidimensional measurement. J Natl Cancer Inst. 1999;91: 523-8.

5. Padhani AR, Husband JE. Are current tumour response criteria relevant for the $21^{\text {st }}$ century? Br J Radiol. 2000;73:1031-3.

6. Suzuki C, Jacobsson H, Hatschek T, Torkzad MR, Bodén K, Eriksson-Alm Y, et al. Radiologic measurements of tumor response to treatment: practical approaches and limitations. Radiographics. 2008;28:329-44.

7. Therasse P, Arbuck SG, Eisenhauer EA, Wanders J, Kaplan RS, Rubinstein L, et al. New guidelines to evaluate the response to treatment in solid tumors. European Organization for Research and Treatment of Cancer, National Cancer Institute of the United States, National Cancer Institute of Canada. J Natl Cancer Inst. 2000;92:205-16.

8. Sargent DJ, Rubinstein L, Schwartz L, Dancey JE, Gatsonis C, Dodd LE, et al. Validation of novel imaging methodologies for use as cancer clinical trial endpoints. Eur J Cancer. 2009;45:290-9.

9. Eisenhauer EA, Therasse P, Bogaerts J, Schwartz LH, Sargent D, Ford R, et al. New response evaluation criteria in solid tumours: revised RECIST guideline (version1.1). Eur J Cancer. 2009;45:228-47.

10. Bogaerts J, Ford R, Sargent D, Schwartz LH, Rubinstein L, Lacombe D, et al. Individual patient data analysis to assess modifications to the RECIST criteria. Eur J Cancer. 2009;45:248-60.

11. Moskowitz CS, Jia X, Schwartz LH, Gonen M. A simulation study to evaluate the impact of the number of lesions measured on response assessment. Eur J Cancer. 2009; 45:300-10

12. Schwartz LH, Mazumdar M, Brown W, Smith A, Panicek DM. Variability in response assessment in solid tumors: effect of number of lesions chosen for measurement. Clin Cancer Res. 2003;9:4318-23.

13. Schwartz LH, Bogaerts J, Ford R, Shankar L, Therasse P, Gwyther S, et al. Evaluation of lymph nodes with RECIST 1.1. Eur J Cancer. 2009:45:261-7.

14. Dancey JE, Dodd LE, Ford R, Kaplan R, Mooney M, Rubinstein L, et al. Recommendations for the assessment of progression in randomized cancer treatment trials. Eur J Cancer. 2009;45:281-9.

15. van Persijn van Meerten EL, Gelderblom H, Bloem JL. RECIST revised: implications for the radiologist. A review article on the modified RECIST guideline. Eur Radiol. 2010;20:1456-67.

16. Sun JM, Ahn MJ, Park MJ, Yi JH, Kim TS, Chung MJ, at al. Accuracy of RECIST 1.1 for non-small cell lung cancer treated with EGFR tyrosine kinase inhibitors. Lung Cancer. 2010;69:105-9.

17. Nishino M, Jackman DM, Hatabu H, Yeap BY, Cioffredi LA, Yap JT, et al. New Response Evaluation Criteria in Solid Tumors (RECIST) guidelines for advanced non-small cell lung cancer: comparison with original RECIST and impact on assessment of tumor response to target therapy. AJR Am J Roentgenol. 2010;195:W221-8.

18. Nishino M, Cardarella S, Jackman DM, Ramaiya NH, Rabin MS, Hatabu H, et al. RECIST 1.1 in NSCLC patients with EGFR mutations treated with EGFR tyrosine kinase inhibitors: comparison with RECIST 1.0. AJR Am J Roentgenol. 2010;201:W64-71.

19. Jang GS, Kim MJ, Ha HI, Kim JH, Kim HS, Ju SB et al. Comparison of RECIST version 1.0 and 1.1 in assessment of tumor response by computed tomography in advanced gastric cancer. Chin J Cancer Res. 2013;25:89-94.

20. Jang HJ, Kim BC, Kim HS, Kim JH, Song HH, Kim JB, et al. Comparison of RECIST 1.0 and RECIST 1.1 on computed tomography in patients with metastatic colorectal cancer. Oncology. 2014;86:117-21. 
21. Ruan M, Shen Y, Chen L, Li M. RECIST 1.1 and serum thyroglobulin measurements in the evaluation of responses to sorafenib in patients with radioactive iodine-refractory differentiated thyroid carcinoma. Oncol Lett. 2013;6:480-6.

22. Fuse N, Nagahisa-Oku E, Doi T, Sasaki T, Nomura S, Kojima T. Effect of RECIST revision on classification of target lesions and overall response in advanced gastric cancer patients. Gastric Cancer. 2013;16:324-8.

23. Piatek CI, Desai BB, Wei-Tsao D, Tang R, Acosta F, Pinski JK, et al. RECIST 1.0 versus 1.1: implications for trial interpretation and design in advanced prostatic cancer. J Clin Oncol. 2011;29 (suppl): abstr2563. 